\title{
Decisiveness indices are semiindices: Addendum
}

\author{
Josep Freixas and Montserrat Pons*
}

\begin{abstract}
In the paper Decisiveness indices are semiindices (Economics Letters 143:13-15, 2016) it was shown that any decisiveness index obtained from an anonymous probability distribution is a semiindex, and that the converse is not true. In this note we characterize the semiindices which are indices of decisiveness.
\end{abstract}

Key words: Game theory; Simple games; Power indices; Semiindices; Decisiveness.

Math. Subj. Class. (2000): 91A12, 91A80. JEL Class.: C71, D72.

\section{Preliminaires}

In this paper we deal with a voting system in which a proposal is put to the vote and it must be either approved or rejected. It is assumed that each voter can only vote "yes" or "no". The appropriate model for this binary voting context is a simple game $(N, \mathcal{W})$, where $N=\{1,2, \ldots, n\}$ denotes the set of voters. A coalition is any subset $S \subseteq N$, and is interpreted as the set of voters which vote "yes". A coalition is winning when its occurrence causes the proposal to be accepted, and $\mathcal{W}$ denotes the set of winning coalitions. For any coalition $S$ we denote $s=|S|$. When the set of voters, $N$, is fixed we will write $\mathcal{W}$ instead of $(N, \mathcal{W})$. A probability distribution over coalitions is a function $P: 2^{N} \rightarrow \mathbb{R}$ such that $P(S) \geq 0$ for all $S \subseteq N$ and $\sum_{S \in 2^{N}} P(S)=1$. $P(S)$ is interpreted as the probability that voters in $S$ vote "yes" and voters is $N \backslash S$ vote "no". Let $\mathcal{P}_{N}$ denote the set of probability distributions over coalitions.

Let $\mathcal{S}_{N}$ be the set of simple games on $N$. A power index on $\mathcal{S}_{N}$ is a map $\psi: \mathcal{S}_{N} \rightarrow \mathbb{R}^{n}$ that assigns to every simple game $\mathcal{W}$ a vector $\psi(\mathcal{W})$ with components $\psi_{i}(\mathcal{W})$ for all $i \in N$. Power indices try to quantify the importance that voters have in the simple game.

Most power indices are based on the notion of cruciality. If $\mathcal{W}$ is a simple game, a player $i \in N$ is said to be crucial for a coalition $S \subseteq N$ with $i \in S$ if $S \in \mathcal{W}$ but $S \backslash\{i\} \notin \mathcal{W}$. We then write $S \in \mathcal{C}_{i}(\mathcal{W})$. In this paper we deal with two families of power indices based on cruciality: semiindices and decisiveness indices. Semiindices were defined on simple games in [6] and the characterization, by means of coefficients, given in the next definition was proved in [2].

\section{Definition 1.1 Semiindex}

A semiindex is a power index on $\mathcal{S}_{N}$ defined, from a vector $\mathbf{p}=\left(p_{1}, \ldots, p_{n}\right)$ such that $\sum_{j=1}^{n} p_{j}\left(\begin{array}{c}n-1 \\ j-1\end{array}\right)=$

*The authors are with the Universitat Politècnica de Catalunya (Campus Manresa), in the Department of Mathematics; e-mails: josep.freixas@upc.edu, montserrat.pons@upc.edu; postal address: EPSEM, Avda. Bases de Manresa, 61-73, E-08242 Manresa, Spain. 
1 and $p_{j} \geq 0$ for all $j \in N$, by the expression

$$
\mathfrak{S}_{i}^{\mathbf{p}}(\mathcal{W})=\sum_{S \in \mathcal{C}_{i}(\mathcal{W})} p_{s}
$$

for any voter $i \in N$.

Well-known examples of semiindices are: the Shapley-Shubik power index $\varphi$ (Shapley and Shubik [5]), for which $p_{s}=1 /\left[n\left(\begin{array}{l}n-1 \\ s-1\end{array}\right)\right]$ for any $s(1 \leq s \leq n)$, and the Banzhaf power index $\beta$ (Banzhaf [1] and Penrose [4]) for which $p_{s}=1 / 2^{n-1}$.

\section{Definition 1.2 Decisiveness index}

$A$ decisiveness index is a power index on $\mathcal{S}_{N}$ defined, from a probability distribution $P \in \mathcal{P}_{N}$, by the expression

$$
\Phi_{i}^{P}(\mathcal{W})=\sum_{S \in \mathcal{C}_{i}(\mathcal{W})}[P(S)+P(S \backslash\{i\})]
$$

for any voter $i \in N$,

The Banzhaf index $\beta$ and the Shapley-Shubik index $\varphi$ are examples of decisiveness indices for particular probability distributions. Indeed, $\beta=\Phi^{P^{*}}$ and $\varphi=\Phi^{P^{* *}}$, where $P^{*}(S)=1 / 2^{n}$ and $P^{* *}(S)=1 /\left[(n+1)\left(\begin{array}{l}n \\ s\end{array}\right)\right]$ for all $S \subseteq N$.

A probability distribution $P \in \mathcal{P}_{N}$ is anonymous if $P(S)=P(T)$ whenever $|S|=|T|$. Let $\mathcal{A}_{N} \subseteq \mathcal{P}_{N}$ denote the set of anonymous probability distributions. If $P \in \mathcal{A}_{N}$ let $P_{s}=P(S)$ for all $S \subseteq N$. Then, $\sum_{s=0}^{n}\left(\begin{array}{l}n \\ s\end{array}\right) P_{s}=1, P_{s} \geq 0$ for all $s=0,1, \ldots, n$ and the decisiveness index in Definition 1.2 reduces to

$$
\Phi_{i}^{P}(\mathcal{W})=\sum_{S \in \mathcal{C}_{i}(\mathcal{W})}\left[P_{s}+P_{s-1}\right]
$$

The following proposition was proved in [3]:

Proposition 1.3 Let $P \in \mathcal{A}_{N}$. Then the decisiveness index $\Phi^{P}$ coincides with the semiindex $\mathfrak{S}^{\mathbf{p}}$ with $\mathbf{p}=\left(p_{1}, \ldots, p_{n}\right)$ defined by $p_{s}=P_{s}+P_{s-1}$ for $s=1, \ldots, n$.

Thus, any decisiveness index given by an anonymous probability distribution is a semiindex. Nevertheless, not every semiindex comes from a decisiveness index. In the following section we identify the semiindices which can be obtained as in Proposition 1.3.

\section{Semiindices given by decisiveness indices}

We introduce a subclass of semiindices which will be proved to be precisely the semiindices which are decisiveness indices.

\section{Definition 2.1 Trimmed semiindex}

Let $\mathfrak{S}^{\mathbf{p}}$ be a semiindex. We say that $\mathfrak{S}^{\mathbf{p}}$ is trimmed if for any odd number $k$ with $1<k \leq n$ and 
for any $s$ with $1 \leq s \leq n-k+1$ the following inequalities are verified:

$$
\sum_{j=0}^{k-1}(-1)^{j} p_{s+j} \geq 0
$$

Notice that for any two trimmed semiindices $\mathfrak{S}^{\mathbf{p}}$ and $\mathfrak{S}^{\mathbf{q}}$, and any $\alpha \in[0,1]$, it is clear that the semiindex constructed from $\alpha p+(1-\alpha) q$, i.e. $\mathfrak{S}^{\alpha \mathbf{p}+(\mathbf{1}-\alpha) \mathbf{q}}$, is also trimmed. Thus the set of trimmed semiindices is a convex set. Observe also that the Banzhaf index $\beta$ and the Shapley-Shubik index $\varphi$ are both trimmed semiindices since their corresponding coefficients fulfil the inequalities in (1). Finally, let us remark that for $n=2$ any semiindex is trimmed. But for any $n \geq 3$ there exist semiindices which are not trimmed, as the following example shows: let $\mathbf{p}=(1 /(2 n-1), 2 /(2 n-$ $1), 0, \ldots, 0)$. Then, taking $k=3$ and $s=1$ in (1) the corresponding inequality is not verified since $p_{1}-p_{2}+p_{3}=-1 /(2 n-1)<0$.

To better illustrate the set of inequalities in (1) we show all of them for $n=7$ :

$$
\begin{array}{rr}
k=3 & p_{1}-p_{2}+p_{3} \geq 0 \\
p_{2}-p_{3}+p_{4} \geq 0 \\
p_{3}-p_{4}+p_{5} \geq 0 \\
p_{4}-p_{5}+p_{6} \geq 0 \\
p_{5}-p_{6}+p_{7} \geq 0 \\
k=5 \quad p_{1}-p_{2}+p_{3}-p_{4}+p_{5} \geq 0 \\
p_{2}-p_{3}+p_{4}-p_{5}+p_{6} \geq 0 \\
p_{3}-p_{4}+p_{5}-p_{6}+p_{7} \geq 0 \\
k=7 \quad p_{3}-p_{2}+p_{4}+p_{5}-p_{6}+p_{7} \geq 0
\end{array}
$$

Finally, notice that if $\mathfrak{S}^{\mathbf{p}}$ is defined from a vector $\mathbf{p}=\left(p_{1}, \ldots, p_{n}\right)$ which verifies either $0 \leq$ $p_{1} \leq \cdots \leq p_{n}$ or $p_{1} \geq \cdots \geq p_{n} \geq 0$ then $\mathfrak{S}^{\mathbf{p}}$ is a trimmed semiindex.

We establish now the main result of the paper.

Theorem 2.2 A semiindex $\mathfrak{S}^{\mathbf{p}}$ is trimmed if and only if there exists $P \in \mathcal{A}_{N}$ such that $\Phi^{P}=\mathfrak{S}^{\mathbf{p}}$.

Proof:

$\Leftarrow)$ Let $P \in \mathcal{A}_{N}$. Then, from Proposition 1.3, the decisiveness index $\Phi^{P}$ defined from $P$ coincides with the semiindex $\mathfrak{S}^{\mathbf{p}}$ with coefficients $p_{s}=P_{s}+P_{s-1}$ for all $s=1, \ldots, n$. To see that $\mathfrak{S}^{\mathbf{p}}$ is trimmed, let $k$ be an odd number with $1<k \leq n$ and $s$ with $1 \leq s \leq n-k+1$. Then,

$$
\sum_{j=0}^{k-1}(-1)^{j} p_{s+j}=\sum_{j=0}^{k-1}(-1)^{j}\left(P_{s+j}+P_{s+j-1}\right)=P_{s-1}+P_{s+k-1} \geq 0
$$

and this proves that $\mathfrak{S}^{\mathbf{p}}$ is trimmed.

$\Rightarrow$ ) Let $\mathbf{p}=\left(p_{1}, \ldots, p_{n}\right)$ define a trimmed semiindex $\mathfrak{S}^{\mathbf{p}}$, i.e., $\mathbf{p}$ verifies the properties established in Definitions 1.1 and 2.1. Taking into account Proposition 1.3 we only need to prove that the linear system $p_{s}=P_{s}+P_{s-1}$ for $s=1, \ldots, n$ has at least a non-negative solution $\left(P_{0}, P_{1}, \ldots, P_{n}\right)$. This system has $n$ equations and $n+1$ variables and it is compatible, with 
one free variable. Taking $P_{0}$ as the free variable, and writing $P_{0}=a$, the solutions of the system can be expressed in terms of $a$, for $s=1, \ldots, n$ :

$$
P_{s}=\sum_{j=0}^{s-1}(-1)^{j} p_{s-j}+(-1)^{s} a .
$$

A value of $a \geq 0$ such that $P_{s} \geq 0$ for $s=1, \ldots, n$ must verify the following inequalities:

$$
\begin{aligned}
& \sum_{j=0}^{r-1}(-1)^{j+1} p_{r-j} \leq a \quad \text { for any } r \text { even }(1 \leq r \leq n) \\
& \sum_{j=0}^{t-1}(-1)^{j} p_{t-j} \geq a \quad \text { for any } t \text { odd }(1 \leq t \leq n) .
\end{aligned}
$$

The existence of such $a$ will be proved if for any even number $r$ and any odd number $t$, both between 1 and $n$, the following inequalities are verified:

$$
\max \left\{0, \sum_{j=0}^{r-1}(-1)^{j+1} p_{r-j}\right\} \leq \sum_{j=0}^{t-1}(-1)^{j} p_{t-j} .
$$

But for $t$ odd $(1 \leq t \leq n)$ it is $0 \leq \sum_{j=0}^{t-1}(-1)^{j} p_{t-j}$ because $\sum_{j=0}^{t-1}(-1)^{j} p_{t-j}=\sum_{j=0}^{t-1}(-1)^{j} p_{1+j}$, and this value is non-negative by the hypothesis (1) if $t>1$ and by the properties of $\mathbf{p}$ established in Definition 1.1 if $t=1$. Thus we only need to prove that

$$
\sum_{j=0}^{r-1}(-1)^{j+1} p_{r-j} \leq \sum_{j=0}^{t-1}(-1)^{j} p_{t-j}
$$

for any even number $r$ and any odd number $t$, both between 1 and $n$.

Let $r$ be even and $t$ be odd $(1 \leq r, t \leq n)$.

- If $r<t$ let $k=t-r$. Clearly, $k$ is odd $(k>1)$ and we can write:

$$
\sum_{j=0}^{t-1}(-1)^{j} p_{t-j}=\sum_{j=0}^{k+r-1}(-1)^{j} p_{k+r-j}=\sum_{j=-k}^{r-1}(-1)^{j+k} p_{r-j}=\sum_{j=0}^{r-1}(-1)^{j+1} p_{r-j}+\sum_{j=1}^{k}(-1)^{j+1} p_{r+j}
$$

so that the inequality (3) is equivalent to

$$
0 \leq \sum_{j=1}^{k}(-1)^{j+1} p_{r+j}=\sum_{j=0}^{k-1}(-1)^{j} p_{r-1+j}
$$

and this inequality is true because of (1).

- If $r>t$ let $k=r-t$. Clearly, $k$ is odd $(k>1)$ and we can write:

$$
\sum_{j=0}^{r-1}(-1)^{j+1} p_{r-j}=\sum_{j=0}^{t-1}(-1)^{j} p_{t-j}+\sum_{j=1}^{k}(-1)^{j} p_{t+j}
$$


so that the inequality (3) is equivalent to

$$
\sum_{j=1}^{k}(-1)^{j} p_{t+j}=\sum_{j=0}^{k-1}(-1)^{j-1} p_{t+j} \leq 0
$$

and this inequality is true because of (1).

Thus, $a$ can have any value between $\max _{\substack{r \text { even } \\ 1 \leq r \leq n}}\left\{0, \sum_{j=0}^{r-1}(-1)^{j+1} p_{r-j}\right\}$ and $\min _{\substack{t \text { odd } \\ 1 \leq t \leq n}}\left\{\sum_{j=0}^{t-1}(-1)^{j} p_{t-j}\right\}$.

Now, with this value of $a$ in (2) and defining $P(S)=P_{s}$ for any $S \subseteq N$ we obtain an anonymous probability distribution over coalitions with $\Phi^{P}=\mathfrak{S}^{\mathbf{p}}$.

\section{Acknowledgements}

This research was partially supported by funds from the Spanish Ministry of Economy and Competitiveness (MINECO) and from the European Union (FEDER funds) under grant MTM201566818-P(MINECO/FEDER).

\section{References}

[1] J.F. Banzhaf. Weighted voting doesn't work: a mathematical analysis. Rutgers Law Review, 19:317-343, 1965.

[2] F. Carreras, J. Freixas, and M.A. Puente. Semivalues as power indices. European Journal of Operational Research, 149:676-687, 2003.

[3] J. Freixas and M. Pons. Decisiveness indices are semiindices. Economics Letters, 143:13-15, 2016.

[4] L.S. Penrose. The elementary statistics of majority voting. Journal of the Royal Statistical Society, 109:53-57, 1946.

[5] L.S. Shapley and M. Shubik. A method for evaluating the distribution of power in a committee system. American Political Science Review, 48:787-792, 1954.

[6] R.J. Weber. Subjectivity in the valuation of games. In O. Moeschlin and D. Pallaschke, editors, Game theory and related topics, pages 129-136. North Holland Publishing Co., Amsterdam, 1979. 